\title{
The Higgs of the Higgs and the diphoton channel
}

\author{
Kristjan Kannike, ${ }^{a}$ Giulio Maria Pelaggi, $^{b}$ Alberto Salvio $^{c}$ and Alessandro Strumia ${ }^{a, b, c}$ \\ ${ }^{a}$ National Institute of Chemical Physics and Biophysics, \\ Rävala 10, 10143 Tallinn, Estonia \\ ${ }^{b}$ Dipartimento di Fisica, Università di Pisa and INFN, \\ Largo Bruno Pontecorvo 3, 56127 Pisa, Italy \\ ${ }^{c}$ CERN, Theory Division, \\ CH-1211 Geneva 23, Switzerland \\ E-mail: Kristjan.Kannike@cern.ch, g.pelaggi@for.unipi.it, \\ alberto.salvio@cern.ch, astrumia@cern.ch
}

ABSTRACT: LHC results do not confirm conventional natural solutions to the Higgs mass hierarchy problem, motivating alternative interpretations where a hierarchically small weak scale is generated from a dimension-less quantum dynamics. We propose weakly and strongly-coupled models where the field that breaks classical scale invariance giving mass to itself and to the Higgs is identified with a possible new resonance within the LHC reach. As an example, we identify such resonance with the $750 \mathrm{GeV}$ diphoton excess recently reported by ATLAS and CMS. Such models can be extrapolated up to the Planck scale, provide Dark Matter candidates and eliminate the SM vacuum instability.

Keywords: Beyond Standard Model, Renormalization Group, Higgs Physics

ArXiv EPrint: 1605.08681 


\section{Contents}

1 Introduction 1

2 Weakly coupled models 2

2.1 Model of type $g$ ) 3

$\begin{array}{lll}2.2 & \text { Model of type } \lambda \text { ) } & 7\end{array}$

3 Strongly coupled models $\quad 10$

$\begin{array}{ll}3.1 \text { The diphoton as a } \mathrm{TC} \eta & 12\end{array}$

$\begin{array}{ll}\text { 3.2 The diphoton as a TC-dilaton or TC } \sigma & 13\end{array}$

4 Conclusions $\quad 14$

$\begin{array}{ll}\text { A RGE for model } g \text { ) } & 15\end{array}$

B RGE for model $\lambda$ ) $\quad 16$

\section{Introduction}

Conventional solutions to the hierarchy problem have been disfavoured by experimental bounds that relegated them to fine-tuned corners of their parameter space. This situation motivated a reconsideration of the hierarchy problem: no physical effects are associated with quadratically divergent corrections to the Higgs squared masses, so maybe we are over-interpreting quantum field theory when we require theories that tame such quadratic divergences.

The hierarchy problem is bypassed if there are no particles much heavier than the Higgs and significantly coupled to it, such that physical corrections to the Higgs mass are naturally small. This heretic context was dubbed 'finite naturalness' in $[1,2]$.

Within the general finite naturalness scenario an interesting sub-set of theories are those described by dimension-less Lagrangians, that assume that massive parameters do not exist at fundamental level, such that all mass scales in nature are generated dynamically, like the QCD scale in the Standard Model. Strictly speaking, finite-naturalness does not necessarily require the absence of masses in the Lagrangian. What makes the dynamical generation of masses attractive is the fact that it can lead to a separation of scales, which depends exponentially on dimensionless couplings. Therefore, this specific setup allows us to justify why the weak scale is many orders of magnitude smaller than the Planck scale, which itself might be generated by dimension-less dynamics, with important implications for inflation [3-11].

Notice that a dimension-less Lagrangian corresponds to a classical scale invariant model. We do not wish to preserve scale invariance at the quantum level because we eventually have to generate the observed scales. When the couplings of the theory are 
small, classical scale invariance is also an approximate symmetry of the model, otherwise it is just the requirement that the masses are dynamically generated (with the motivations stated above).

Various models where the weak scale arises in this way have been proposed. They can be classified in two categories:

1. The weak scale is the scale where a scalar quartic coupling $\lambda$ runs negative, inducing vacuum expectation values à la Coleman-Weinberg [12-38].

2. The weak scale is the scale where a gauge coupling $g$ runs to non-perturbative values, inducing condensates [39-43].

It is interesting to ask whether these theoretical frameworks could lead to something visible at the LHC. A particularly clean channel, which has therefore a great discovery potential, is the one with two photons in the final state. We therefore study models where one of the required new particles can be identified with a new resonance $\digamma$ decaying into two photons (diphoton). As an example, we treat in some detail the case in which such resonance is identified with the recent diphoton excess at $750 \mathrm{GeV}$ reported by ATLAS and CMS $[44,45]$. Other numerical choices are of course possible and our work will remain valuable even if such excess will turn out to be a statistical fluctuation.

Connections with conventional solutions to the hierarchy problem have been explored by authors who tried to identify $\digamma$ with one or another supersymmetric particle [46-67] or with resonances of composite Higgs scenarios [46, 68-70].

References $[71,72]$ tried to incorporate the diphoton hinted by ATLAS and CMS in the weakly-coupled framework; however, in both cases couplings are so large that the attempted models hit Landau poles just above the weak scale, such that no hierarchy is dynamically generated. ${ }^{1}$

In section 2 we present weakly-coupled dimensionless models where $\digamma$ is the field that dynamically generates the weak scale, while running down from the Planck scale. In section 3 we present strongly coupled dimension-less models, where, among other things, the diphoton excess is reproduced. In section 4 we present our conclusions.

\section{Weakly coupled models}

Various extensions of the SM where the weak scale is generated $\grave{a}$ la Coleman-Weinberg have been proposed in the literature. They can be divided into two main categories, depending on which correction renormalises a quartic coupling $\lambda_{\digamma}$ down to negative values at low energy, such that the dimension-less potential $\lambda_{\digamma} \digamma^{4}$ develops a minimum: either

$\lambda)$ corrections due to other scalar quartics [13-32]; or

g) corrections due to a gauge coupling [12,33-38].2

\footnotetext{
${ }^{1}$ Furthermore [71] also contain some explicit mass term. The model in [73, 74] can be extrapolated up to infinite energy, but it employs explicit mass terms. It is possible that these mass terms could be generated at a scale much higher than the weak scale. But finding an example of this sort goes beyond the scope of the present paper.

${ }^{2}$ Yukawa couplings have the opposite effect of making a quartic larger at low energy.
} 


\begin{tabular}{|cc|cccc|}
\hline name & spin & $\mathrm{SU}(3)_{c}$ & $\mathrm{SU}(2)_{L}$ & $\mathrm{U}(1)_{Y}$ & $\mathrm{SU}(N)$ \\
\hline$X$ & 0 & 1 & 1 & 0 & 1 \\
$S$ & 0 & 1 & 1 & 0 & $N$ \\
\hline $\mathcal{U}$ & $1 / 2$ & $\overline{3}$ & 1 & $-2 / 3$ & $N$ \\
$\mathcal{U}^{c}$ & $1 / 2$ & 3 & 1 & $+2 / 3$ & $\bar{N}$ \\
\hline
\end{tabular}

Table 1. Beyond the Standard Model field content of the model of type $g$ ) of section 2.1.

Models of type $\lambda$ ) are more problematic than models of type $g$ ), because a non-abelian gauge coupling $g$ can be sizeable without implying nearby Landau poles, while a sizeable quartic coupling drives itself to larger values at higher energies. Models of type $\lambda$ ) in the literature mitigate this effect by having a large number $N$ of smaller quartics, and we will follow this strategy. We present in section 2.1 (section 2.2) models of type $g$ ) (of type $\lambda$ ) where the Coleman-Weinberg field is the diphoton, and where the RGE can be extrapolated up to the Planck scale, such that a large hierarchy is dynamically generated.

Before starting, we mention a broader - but less interesting — class of scale-invariant models, where the diphoton is added as an ad-hoc extra field that does not play a key role in the dynamical generation of the weak scale. Roughly, one can choose any one of the $N$ diphoton models proposed in the literature, and any one of the $M$ models that dynamically generated the weak scale, and combine them into $N \times M$ models.

For example, there is no obstacle in combining the dimension-less model in [12] (an extra $\mathrm{SU}(2)$ gauge interaction with an extra scalar doublet $S$, that acquires a vacuum expectation value through the Coleman-Weinberg mechanism) with the 'diphoton everybody's model' [46] (the diphoton is an extra scalar singlet $\digamma$ coupled to extra charged scalars $X$ or fermions $\psi$ ). The $\mathrm{SU}(2)$ massive vectors are DM candidates [12].

What are the generic features of this class of ad-hoc models? The new charged particles cannot be arbitrarily heavier than the Higgs mass $M_{h}$, otherwise they contribute to it unnaturally $[1,2]$ :

$$
\begin{array}{ll}
M_{\psi} \lesssim 4 \pi M_{h} / g_{1,2}^{2} & \text { for fermions, } \\
M_{X} \lesssim M_{h} / g_{1,2}^{2} & \text { for scalars, }
\end{array}
$$

where $g_{1,2}$ are the electroweak gauge couplings. The measured diphoton rate indeed suggests new particles with sub- TeV mass [46]. Furthermore, in the context of finite naturalness, the existence of extra colored fermions below a few $\mathrm{TeV}$ is necessary if the QCD $\theta$ problem is solved by an KSVZ axion model [1,2].

We now propose more interesting - but more constrained - models where the diphoton $\digamma$ is the particle that dynamically acquires the vacuum expectation value that induces the electroweak scale.

\subsection{Model of type $g$ )}

We extend the SM by adding an extra gauge group $\mathrm{SU}(N)$ and the extra fields listed in table 1: two scalars $S$ and $X$, a quark $\mathcal{U}$ with the same quantum numbers of the SM 
right-handed up quarks that fill a $N$ of $\mathrm{SU}(N)$, plus the conjugated fermion $\mathcal{U}^{c}$ in order to form a vector-like quark. The dimension-less Yukawa couplings are

$$
\mathscr{L}_{Y}=\mathscr{L}_{Y}^{\mathrm{SM}}+\left(y_{S} S \mathcal{U}^{c} U+y_{X} X \mathcal{U} \mathcal{U}^{c}+\text { h.c. }\right)
$$

where $U$ is the right-handed up quark of the SM. The dimension-less potential of the theory is

$$
V(H, S, X)=\lambda_{H}|H|^{4}+\lambda_{X} X^{4}+\lambda_{S}|S|^{4}-\lambda_{H S}|H|^{2}|S|^{2}-\lambda_{H X}|H|^{2} X^{2}-\lambda_{S X}|S|^{2} X^{2} .
$$

The tree-level potential is positive, $V \geq 0$, when the quartic couplings satisfy [75]

$$
\left\{\begin{array}{l}
\lambda_{H} \geq 0, \quad \lambda_{S} \geq 0, \quad \lambda_{X} \geq 0 \\
\bar{\lambda}_{H S} \equiv-\lambda_{H S}+2 \sqrt{\lambda_{H} \lambda_{S}} \geq 0 \\
\bar{\lambda}_{H X} \equiv-\lambda_{H X}+2 \sqrt{\lambda_{H} \lambda_{X}} \geq 0 \\
\bar{\lambda}_{S X} \equiv-\lambda_{S X}+2 \sqrt{\lambda_{S} \lambda_{X}} \geq 0 \\
-\sqrt{\lambda_{H}} \lambda_{X S}-\sqrt{\lambda_{S}} \lambda_{H X}-\sqrt{\lambda_{X}} \lambda_{H S}+2 \sqrt{\lambda_{H} \lambda_{S} \lambda_{X}}+\sqrt{\bar{\lambda}_{H S} \bar{\lambda}_{H X} \bar{\lambda}_{S X}} \geq 0
\end{array}\right.
$$

The RGEs of the model are listed in appendix A. Notice that $\lambda_{H S}$ is unavoidably generated from the Yukawa couplings, if the top is the quark mostly coupled to the new states $\mathcal{U}$.

Masses. When the field $S$ dynamically acquires a vacuum expectation value breaking $\mathrm{SU}(N) \rightarrow \mathrm{SU}(N-1)$ (to nothing if $N=2[12]$ ), $H$ and $X$ too can acquire vacuum expectation values, in view of their $\lambda_{H S}$ and $\lambda_{S X}$ quartic couplings to $S$. In the unitary gauge there are three physical scalars:

$$
S=\left(v_{S}+\frac{s}{\sqrt{2}}, 0, \ldots\right), \quad X=v_{X}+x, \quad H=\left(v+\frac{h}{\sqrt{2}}, 0\right) .
$$

We assume that $\lambda_{H S}$ and $\lambda_{H X}$ are negligible, and that scale invariance gets broken when the RGE running of $\lambda_{S}$, dominated by the gauge coupling $g$, violates the condition $\bar{\lambda}_{S X} \geq 0$ of eq. (2.3). We can approximate the one loop potential by inserting a running

$$
\lambda_{S}(S)=\frac{\lambda_{S X}^{2}}{4 \lambda_{X}}+\beta_{\lambda_{S}} \ln \frac{|S|}{S_{*}}
$$

in the tree-level potential of eq. (2.3). Here $S_{*}$ is the scale at which the stability condition $\bar{\lambda}_{S X} \geq 0$ is violated. For $|S|$ around $S_{*}$ there is an approximately flat direction, which is lifted by quantum correction (the $\log$ term in eq. (2.6)) as illustrated in figure 1. This generates the following absolute minimum of the potential

$$
v_{S}=\frac{S_{*}}{e^{1 / 4}}, \quad v_{X}=v_{S} \sqrt{\frac{\lambda_{S X}}{2 \lambda_{X}}}
$$

which is visible in figure 1 . There is another absolute minimum with the sign of $v_{X}$ switched, but we assume $v_{X}>0$ without loss of generality. The scalar mass matrix at the minimum in the $(x, s)$ basis is

$$
2 v_{S}^{2}\left(\begin{array}{cc}
2 \lambda_{S X} & -\sqrt{\lambda_{S X}^{3} / \lambda_{X}} \\
-\sqrt{\lambda_{S X}^{3} / \lambda_{X}} & \beta_{\lambda_{S}}+\lambda_{S X}^{2} / 2 \lambda_{X}
\end{array}\right) .
$$




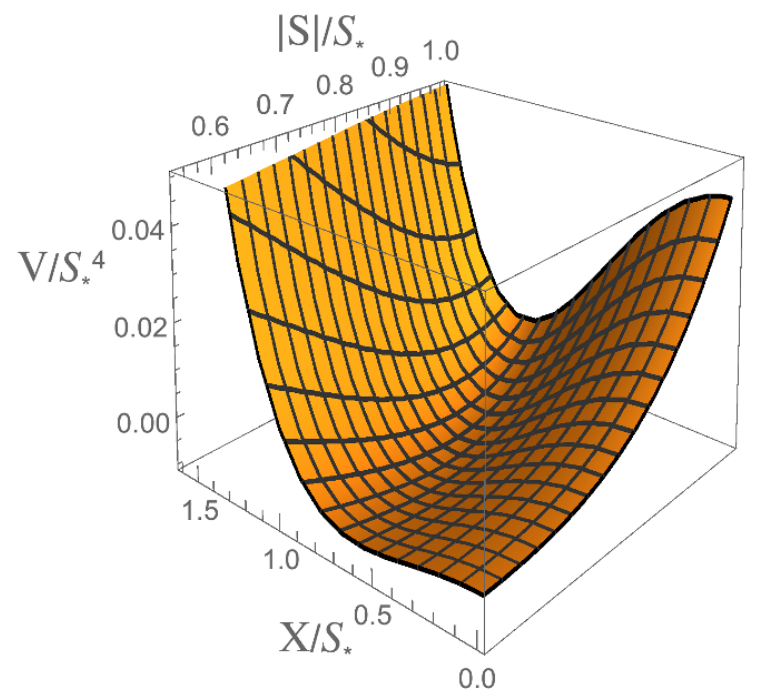

Figure 1. Potential defined in eq. (2.3) for $\lambda_{S}$ chosen as in eq. (2.6) and $H=0$. We set $\beta_{\lambda_{S}} \simeq 0.12$, $\lambda_{X} \simeq 0.030$ and $\lambda_{S X} \simeq 0.071$.

Neglecting the small mixing with the Higgs, the mass eigenstates are the diphoton $\digamma$ and a similar heavier scalar $\digamma^{\prime}$

$$
\left\{\begin{array}{l}
\digamma=s \cos \theta+x \sin \theta \\
\digamma^{\prime}=x \cos \theta-s \sin \theta
\end{array} \quad \text { where } \quad \tan 2 \theta=\frac{4 \lambda_{S X}^{3 / 2} \lambda_{X}^{1 / 2}}{4 \lambda_{X} \lambda_{S X}-\lambda_{S X}^{2}-2 \lambda_{X} \beta_{\lambda_{S}}} .\right.
$$

Their masses are

$$
M_{\digamma, \digamma^{\prime}}^{2}=\frac{v_{S}^{2}}{2 \lambda_{X}}\left[A \mp \sqrt{A^{2}-32 \beta_{\lambda_{S}} \lambda_{X}^{2} \lambda_{S X}}\right], \quad A \equiv 4 \lambda_{X} \lambda_{S X}+\lambda_{S X}^{2}+2 \lambda_{X} \beta_{\lambda_{S}} .
$$

The Higgs mass is $M_{h}^{2} \simeq 2\left(\lambda_{H S} v_{S}^{2}+\lambda_{H X} v_{X}^{2}\right)$. The mixing angle between the higgs and the diphoton is experimentally constrained to be small $[46,76]$

$$
\left|\sin \theta_{h \digamma}\right| \lesssim 0.015 \sqrt{\Gamma_{\gamma \gamma} / 10^{-6} M_{\digamma}} .
$$

In the present model such mixing angle is of order $\theta_{h \digamma} \sim M_{h}^{2} v / M_{\digamma}^{2} v_{S, X}$, which is below its experimental bound provided that $v_{S, X} \gtrsim 500 \mathrm{GeV}$.

Coming to fermion masses, $\mathcal{U}$ and $\mathcal{U}^{c}$ split into $N-1$ vector-like up quarks with mass $M_{N-1}=y_{X} v_{X}$ and into one with mass $M_{1}=\sqrt{\left(y_{S} v_{S}\right)^{2}+\left(y_{X} v_{X}\right)^{2}}$, having neglected smaller electroweak contributions. Recasting LHC searches for similar objects [77], we estimate a bound $M \gtrsim 1.2(1.3) \mathrm{TeV}$ on their masses for $N=1(5)$.

Diphoton rate. The $\mathcal{U}$ heavy quarks mediate diphoton decays into SM vectors. In the limit where the particles $\wp$ that induce loop decays of a generic scalar $\varphi$ into SM vectors are much heavier than the energy involved in the decay, their contribution to the decay amplitude is related to their contributions to the $\beta$ function coefficients $\Delta b_{i}^{\wp}$ of the SM 
gauge couplings as $[78,79]$

$$
\mathscr{L}_{\text {eff }}=\sum_{i, \wp} \Delta b_{i}^{\wp} \frac{\alpha_{i}}{8 \pi}\left(F_{\mu \nu}^{i}\right)^{2} \ln \frac{M_{\wp}(\varphi)}{M_{\wp}}
$$

where $M_{\wp}(\varphi)$ is the $\wp$ mass for a generic vev of $\varphi$. In our model $\varphi=\{x, s\}=\left\{\digamma, \digamma^{\prime}\right\}$ and the loop particles $\wp$ are the quark triplets with mass $M_{N-1}=y_{X} X\left(\Delta b_{\mathrm{em}}=16 / 9\right)$ and with mass $M_{1}=\sqrt{y_{X} X^{2}+y_{S}|S|^{2}}\left(\Delta b_{\mathrm{em}}=(N-1) 16 / 9\right)$. The other $\Delta b_{i}$ coefficients are given by eqs. (A.1i) and (A.1k). So

$$
\mathscr{L}_{\text {eff }}^{\gamma}=\frac{\alpha_{\mathrm{em}}}{9 \pi} F_{\mu \nu}^{2}\left[\sqrt{2} s y_{S} \frac{\sqrt{M_{1}^{2}-M_{N-1}^{2}}}{M_{1}^{2}}+2 x y_{X} \frac{(N-1) M_{1}^{2}+M_{N-1}^{2}}{M_{1}^{2} M_{N-1}}\right] .
$$

Rotating to the mass eigenstates one finds the $\digamma$ width into $\gamma \gamma$ :

$$
\begin{gathered}
\frac{\Gamma(\digamma \rightarrow \gamma \gamma)}{M_{\digamma}}=\frac{4 M_{\digamma}^{2} \alpha_{\mathrm{em}}^{2}}{81 \pi^{3}}\left(\frac{\sqrt{2} y_{S}^{2} v_{S} v_{X} \cos \theta+2\left(N y_{X}^{2} v_{X}^{2}+(N-1) y_{S}^{2} v_{S}^{2}\right) \sin \theta}{v_{X}\left(y_{S}^{2} v_{S}^{2}+y_{X}^{2} v_{X}^{2}\right)}\right)^{2}, \\
\frac{\Gamma\left(\digamma^{\prime} \rightarrow \gamma \gamma\right)}{M_{\digamma^{\prime}}}=\frac{4 M_{\digamma^{\prime}}^{2} \alpha_{\mathrm{em}}^{2}}{81 \pi^{3}}\left(\frac{\sqrt{2} y_{S}^{2} v_{S} v_{X} \sin \theta-2\left(N y_{X}^{2} v_{X}^{2}+(N-1) y_{S}^{2} v_{S}^{2}\right) \cos \theta}{v_{X}\left(y_{S}^{2} v_{S}^{2}+y_{X}^{2} v_{X}^{2}\right)}\right)^{2} .
\end{gathered}
$$

In the limit of small $\beta_{\lambda_{S}}$ the pseudo-Goldstone of scale invariance is the lighter state $\digamma$ :

$$
M_{\digamma}^{2} \simeq \frac{2 v_{S}^{2} \beta_{\lambda_{S}}}{1+\lambda_{S X} / 4 \lambda_{X}} \ll M_{\digamma^{\prime}}^{2} \simeq 4 v_{S}^{2} \lambda_{S X}\left(1+\frac{\lambda_{S X}}{4 \lambda_{X}}\right), \quad \tan \theta=\frac{v_{X}}{\sqrt{2} v_{S}} \simeq \sqrt{\frac{\lambda_{S X}}{2 \lambda_{X}}}
$$

and eq. (2.14a) reduces to

$$
\frac{\Gamma(\digamma \rightarrow \gamma \gamma)}{M_{\digamma}} \simeq \frac{8 N^{2} M_{\digamma}^{2} \alpha_{\mathrm{em}}^{2}}{81 \pi^{3}\left(v_{S}^{2}+v_{X}^{2} / 2\right)}
$$

In the less relevant opposite limit of small $\lambda_{S X}$ (and thereby $v_{X} \ll v_{S}$ ) the pseudoGoldstone of scale invariance is the heavier scalar $\digamma^{\prime}$ :

$$
M_{\digamma}^{2} \simeq 4 v_{S}^{2} \lambda_{S X} \ll M_{\digamma^{\prime}}^{2} \simeq 2 v_{S}^{2} \beta_{\lambda_{S}}, \quad \tan \theta \simeq \beta_{\lambda_{S}} \sqrt{\frac{\lambda_{X}}{\lambda_{S X}^{3}}}
$$

and eq. (2.14b) reduces to

$$
\frac{\Gamma\left(\digamma^{\prime} \rightarrow \gamma \gamma\right)}{M_{\digamma^{\prime}}} \simeq \frac{8 M_{\digamma^{\prime}}^{2} \alpha_{\mathrm{em}}^{2}}{81 \pi^{3} v_{S}^{2}}
$$

Going beyond the approximation of eq. (2.12) requires computing Feynman diagrams with two different masses in the loop. In the limit $y_{S}=0$, such that $M_{1}=M_{N-1}$, the full expression is

$$
\frac{\Gamma(\digamma \rightarrow \gamma \gamma)}{M_{\digamma}}=\frac{4 N^{2} M_{1}^{2} \alpha_{\mathrm{em}}^{2} y_{X}^{2} \sin ^{2} \theta}{9 \pi^{3} M_{\digamma}^{2}}\left|\mathcal{S}\left(\frac{4 M_{1}^{2}}{M_{\digamma}^{2}}\right)\right|^{2}
$$

where the loop function $\mathcal{S}$ is

$$
\mathcal{S}(x)=1+(1-x) \arctan ^{2}\left(\frac{1}{\sqrt{x-1}}\right) \stackrel{x \gg 1}{\simeq} \frac{2}{3 x} .
$$

In the limit $M_{1} \gg M_{\digamma}$ eq. (2.19) reduces to (2.16). 

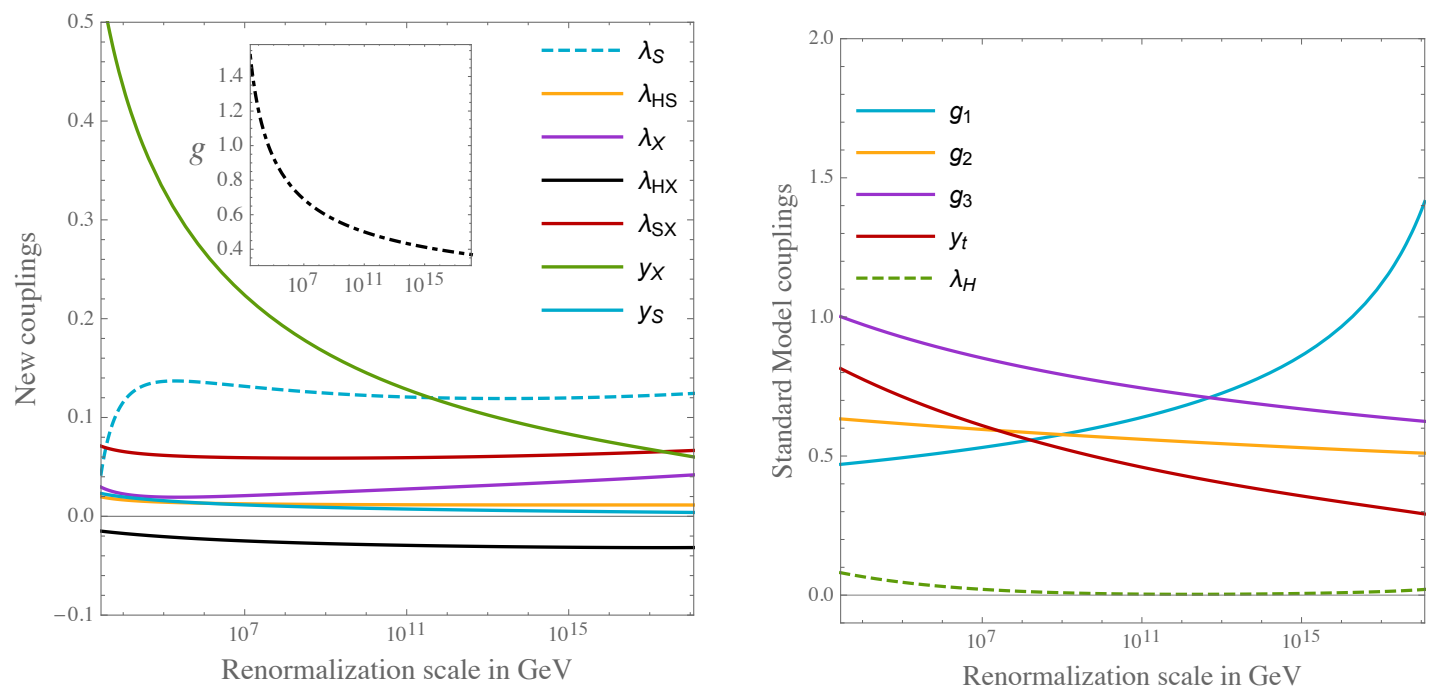

Figure 2. Running of the couplings for central values for the SM parameters and for $N=5$, $g\left(S_{*}\right) \simeq 1.5, v_{S} \simeq 2.2 \mathrm{TeV}, \lambda_{H X}\left(S_{*}\right) \simeq-0.015, \lambda_{S X}\left(S_{*}\right) \simeq 0.071, \lambda_{X}\left(S_{*}\right) \simeq 0.030, y_{S}\left(S_{*}\right)=0.023$ and $y_{X}\left(S_{*}\right)=0.53$.

Numerical example. By performing a global fit of run 1 and run 2 ATLAS and CMS data assuming a narrow width and dominant $g g \rightarrow \digamma$ production we find the diphoton rate $\sigma(p p \rightarrow \digamma \rightarrow \gamma \gamma)=(2.8 \pm 0.7) \mathrm{fb}$ at $s=(13 \mathrm{TeV})^{2}$, which is reproduced for

$$
\frac{\Gamma_{\gamma \gamma}}{M_{\digamma}}=\frac{s \sigma(p p \rightarrow \gamma \gamma)}{K_{g g} C_{g g}}=(3.8 \pm 0.9) 10^{-7}
$$

where $K_{g g} C_{g g}=1.5 \times 2140$ are partonic factors [46].

In the limit of small $\beta_{\lambda_{S}}$ the diphoton mass and decay width are reproduced for

$$
v_{S}=N \frac{310 \mathrm{GeV}}{\sqrt{1+\tan ^{2} \theta}} \sqrt{\frac{10^{-6} M_{\digamma}}{\Gamma_{\gamma \gamma}}}, \quad \beta_{\lambda_{S}}=\frac{5.9}{N^{2}}\left(1+\tan ^{2} \theta\right)^{2} \frac{\Gamma_{\gamma \gamma}}{10^{-6} M} .
$$

Even for $N=2$ this corresponds to a perturbative value of $g$, with $N g^{2} /(4 \pi)^{2}$ becoming smaller at larger $N$.

In figure 2 we provide a numerical example with $M_{\digamma}=750 \mathrm{GeV}, M_{\digamma^{\prime}} \simeq 1.7 \mathrm{TeV}, \Gamma_{\gamma \gamma} \approx$ $3.5 \times 10^{-7} M_{\digamma}, M_{1} \simeq M_{N-1} \approx 1.3 \mathrm{TeV}$. There are no Landau poles at energies much smaller than the Planck scale, and the stability conditions of eq. (2.4) are violated only at low energy, when the desired Coleman-Weinberg mechanism takes place. We assumed central values for the SM parameters, and the Higgs quartic $\lambda_{H}$ remains positive up to the Planck scale, unlike in the SM: the new dynamics eliminated the SM vacuum instability [80-82]. ${ }^{3}$

\section{$2.2 \quad$ Model of type $\lambda$ )}

Models of type $\lambda$ ) - those where the diphoton quartic is driven negative by RGE effects of other quartics - need a multiplicity of $N$ scalars in order to avoid Landau poles nearby by

\footnotetext{
${ }^{3}$ Needless to say, new physics at the Planck scale can give new sources of vacuum decay faster than the SM instability scale and faster than the diphoton lifetime. The first part of this statement was strongly emphasised in [83]. For other diphoton models addressing the vacuum instability of the SM see [84-90].
} 


\begin{tabular}{|cc|cccc|}
\hline name & spin & $\mathrm{SU}(3)_{c}$ & $\mathrm{SU}(2)_{L}$ & $\mathrm{U}(1)_{Y}$ & $\mathrm{SU}(N)$ \\
\hline$X$ & 0 & 3 & 1 & $Y$ & $N$ \\
$S$ & 0 & 1 & 1 & 0 & 1 \\
\hline $\mathcal{N}$ & $1 / 2$ & 1 & 1 & 0 & $N$ \\
$\mathcal{N}^{c}$ & $1 / 2$ & 1 & 1 & 0 & $\bar{N}$ \\
\hline
\end{tabular}

Table 2. Beyond the Standard Model field content of the model of type $\lambda$ ) of section 2.2.

sharing the needed relatively large quartics. Some diphoton models introduce a multiplicity of $N$ states for a different reason: in order to mediate a sufficiently large $\digamma \rightarrow \gamma \gamma$ rate. We thereby identify the charged particles that mediate $\digamma \rightarrow \gamma \gamma$ with the scalars that drive the diphoton quartic to negative values.

We now show that a successful model of this type is obtained by considering the massless limit of the diphoton model proposed in [91], where the multiplicity of $N$ states is justified by adding an extra gauge group $\mathrm{SU}(N)$ with gauge coupling $g$. The model employs the field content listed in table 2. The three scalars are: the SM Higgs doublet $H$, a neutral singlet $S$ that will contain the diphoton $\digamma$, and a charged and colored scalar $X$ in the fundamental $N$ of $\mathrm{SU}(N)$, that mediates $\digamma \rightarrow \gamma \gamma, g g$ at one loop.

Including the most generic dimension-less quartic couplings, the scalar potential of the model is:

$$
\begin{aligned}
V(H, S, X)= & \lambda_{H}|H|^{4}-\lambda_{H S}|H|^{2} S^{2}+\lambda_{S} S^{4}+\lambda_{H X}|H|^{2}|X|^{2} \\
& +\lambda_{X S} S^{2}|X|^{2}+\lambda_{X} \operatorname{Tr}\left(X X^{\dagger}\right)^{2}+\lambda_{X}^{\prime} \operatorname{Tr}\left(X X^{\dagger} X X^{\dagger}\right) .
\end{aligned}
$$

The tree-level potential satisfies $V \geq 0$ when the quartic couplings satisfy [75]

$$
\left\{\begin{array}{l}
\lambda_{S}>0, \quad \lambda_{H}>0, \quad \lambda_{X}+\alpha \lambda_{X}^{\prime} \geq 0 \\
\bar{\lambda}_{H S} \equiv-\lambda_{H S}+2 \sqrt{\lambda_{H} \lambda_{S}} \geq 0 \\
\bar{\lambda}_{H X} \equiv \lambda_{H X}+2 \sqrt{\lambda_{H}\left(\lambda_{X}+\alpha \lambda_{X}^{\prime}\right)} \geq 0 \\
\bar{\lambda}_{X S} \equiv \lambda_{X S}+2 \sqrt{\lambda_{S}\left(\lambda_{X}+\alpha \lambda_{X}^{\prime}\right)} \geq 0 \\
\sqrt{\lambda_{H}} \lambda_{X S}+\sqrt{\lambda_{S}} \lambda_{H X}-\sqrt{\lambda_{X}+\alpha \lambda_{X}^{\prime}} \lambda_{H S}+2 \sqrt{\lambda_{H} \lambda_{S}\left(\lambda_{X}+\alpha \lambda_{X}^{\prime}\right)}+\sqrt{\bar{\lambda}_{H S} \bar{\lambda}_{H X} \bar{\lambda}_{X S}} \geq 0
\end{array}\right.
$$

for $\alpha=1$ and for $\alpha=1 / N_{c}$ (for $N \geq N_{c}=3$ ), which are the extremal values of

$$
\alpha=\operatorname{Tr}\left(X X^{\dagger} X X^{\dagger}\right) / \operatorname{Tr}\left(X X^{\dagger}\right)^{2} .
$$

A Coleman-Weinberg minimum is generated when the RGE running of the quartics crosses the boundary of one of these conditions. In practice, we are interested in the case where $\lambda_{S}$ becomes negative while running to low energy, while $\lambda_{H S}>0$ (such that the Higgs too acquires a vev) and $\lambda_{H X}>0$ (such that $X$ does not acquire any vev).

Furthermore, the model contains $N_{f}$ extra fermions $\mathcal{N} \oplus \mathcal{N}^{c}$ with no SM gauge interactions and in the $N \oplus \bar{N}$ representation of $\mathrm{SU}(N)$. Such fermions receive mass from 
$S \mathcal{N N}^{c}$ Yukawa couplings. The lightest among them and among the $\mathrm{SU}(N)$ vectors are Dark Matter candidates. The Yukawa couplings $X^{*} U \mathcal{N}$ (allowed if $Y=2 / 3$ ) induces $X$ decays into SM up quarks and $N$. Recasting LHC searches for similar objects [77], we estimate a bound $M_{X} \gtrsim 1.0(1.2) \mathrm{TeV}$ on their masses for $N=3$ (10). We assume that such extra Yukawa couplings are small enough that we can neglect their contributions to the RGE. In the presence of these extra fermions, the RGE acquire infra-red fixed points which allow the model to be RGE-extrapolated up to the Planck scale with a stable potential.

The RGE of the model are listed in appendix B. They allow $\lambda_{H S}$ and $\lambda_{X S}$ to be naturally small, while $\lambda_{H X} \sim g_{1}^{4}, \lambda_{S} \sim g^{4}, \lambda_{H} \sim g_{2}^{4}$.

Masses. The potential at one loop order can be approximated by inserting a running $\lambda_{S}$ in the tree-level potential of eq. (2.24):

$$
\lambda_{S} \simeq \beta_{\lambda_{S}} \ln \frac{S}{S_{*}},
$$

where $S_{*}$ is the scale below which $\lambda_{S}$ becomes negative and $\beta_{\lambda_{S}}$ is given in eq. (B.1a). We can here neglect the running of the other couplings. Expanding the scalars as

$$
S=w+s, \quad H=\frac{1}{\sqrt{2}}\left(\begin{array}{c}
0 \\
v+h
\end{array}\right)
$$

the effective potential is minimised by

$$
w=S_{*} \exp \left[-\frac{1}{4}+\frac{\lambda_{H S}^{2}}{4 \lambda_{H} \beta_{\lambda_{S}}}\right], \quad v=w \sqrt{\frac{\lambda_{H S}}{\lambda_{H}}} .
$$

The scalar mass matrix at the minimum in the $(h, s)$ basis is

$$
2 v^{2}\left(\begin{array}{cc}
\lambda_{H} & -\sqrt{\lambda_{H} \lambda_{H S}} \\
-\sqrt{\lambda_{H} \lambda_{H S}} & \lambda_{H S}+2 \beta_{\lambda_{S}} \lambda_{H} / \lambda_{H S}
\end{array}\right) .
$$

The mass eigenstates are the physical higgs and the diphoton $\digamma$. In the limit of small $\epsilon \equiv \lambda_{H S}^{2} / 2 \lambda_{H} \beta_{\lambda_{S}}$, which corresponds to a Higgs mass smaller than the diphoton mass, the mass eigenvalues are

$$
M_{h}^{2} \simeq 2 v^{2} \lambda_{H}(1-\epsilon+\cdots), \quad M_{\digamma}^{2} \simeq 4 w^{2} \beta_{\lambda_{S}}(1+\epsilon+\cdots) .
$$

The diphoton mass $M_{\digamma}$ is suppressed by a one loop factor because the diphoton is identified here with the pseudo-Goldstone boson of scale invariance. The $h / \digamma$ mixing angle

$$
\theta_{h \digamma} \simeq \frac{M_{h}^{2}}{M_{\digamma}^{2}} \frac{v}{w}
$$

is below the experimental bound of eq. (2.11) for $w \gtrsim 500 \mathrm{GeV}$. Finally, $X$ acquires the mass $M_{X} \simeq w \sqrt{\lambda_{X S}}$, the extra fermions receive mass $M_{\mathcal{N}}=y_{S} w$ from $y_{S} S \mathcal{N N}^{c}$ Yukawa couplings, and the SM particles acquire the usual masses through the Higgs vacuum expectation value. 
Diphoton rate. The $\Gamma_{\gamma \gamma} \equiv \Gamma(\digamma \rightarrow \gamma \gamma)$ rate is given by

$$
\frac{\Gamma_{\gamma \gamma}}{M_{\digamma}}=\frac{9 N^{2} \alpha_{\mathrm{em}}^{2} Y^{4}}{256 \pi^{3}}\left|\frac{w \lambda_{X S} M_{\digamma}}{M_{X}^{2}} F\left(\frac{4 M_{X}^{2}}{M_{\digamma}^{2}}\right)\right|^{2}
$$

where the loop function $F$ is

$$
F(x)=x\left[x \arctan ^{2}\left(\frac{1}{\sqrt{x-1}}\right)-1\right] \stackrel{x \rightarrow \infty}{=} \frac{1}{3} .
$$

In the limit $M_{X} \gg M_{\digamma} / 2$ the $\Gamma_{\gamma \gamma}$ rate does not depend on $\lambda_{H S}$, as can be understood using the Low Energy Theorem of eq. (2.12), taking into account that, in the present model, $\wp=X$ with $M_{X}(\digamma) / M_{X}=\digamma / w+\cdots$.

As usual a large multiplicity $N$ enhances $\Gamma_{\gamma \gamma}$; in our context it also enhances $M_{\digamma}$. In order to reproduce desired values of $M_{h}=125 \mathrm{GeV}, M_{\digamma}=750 \mathrm{GeV}, M_{X}$ and $\Gamma_{\gamma \gamma}$, the model parameters are fixed to the following values

$$
\begin{array}{ll}
\lambda_{X S} \approx 0.24 Y^{4}\left(\frac{\mathrm{TeV}}{M_{X}}\right)^{6}\left(\frac{10^{-6}}{\Gamma_{\gamma \gamma} / M_{\digamma}}\right), & w \approx \frac{2.0 \mathrm{TeV}}{Y^{2}}\left(\frac{M_{X}}{\mathrm{TeV}}\right)^{4}\left(\frac{\Gamma_{\gamma \gamma} / M_{\digamma}}{10^{-6}}\right)^{1 / 2}, \\
\lambda_{H S} \approx 0.002 Y^{4}\left(\frac{10^{-6}}{\Gamma_{\gamma \gamma} / M_{\digamma}}\right), & N \approx \frac{30}{Y^{4}}\left(\frac{M_{X}}{\mathrm{TeV}}\right)^{4}\left(\frac{\Gamma_{\gamma \gamma} / M_{\digamma}}{10^{-6}}\right) .
\end{array}
$$

at leading order in $M_{h} / M_{\digamma} \ll 1$, and neglecting higher order corrections such as the running of the coupling constants between $M_{\digamma}$ and $S_{*}$. We see that $N$ is large (tens) for $Y=2 / 3$ and depends strongly on $Y$, such that a small $N$ is obtained for $Y=4 / 3$ or $5 / 3$.

Numerical examples. In figure 3 we provide two numerical examples with $M_{\digamma}=$ $750 \mathrm{GeV}$, with no Landau poles at energies much smaller than the Planck scale and with all the stability conditions of eq. (2.24) satisfied. We assumed central values for the SM parameters, and the Higgs quartic $\lambda_{H}$ remains positive up to the Planck scale unlike in the SM.

In the upper example we have $Y=2 / 3$ and $M_{X} \approx 1.2 \mathrm{TeV}$ The example leads to $\Gamma_{\gamma \gamma} / M_{\digamma} \simeq 3.1 \times 10^{-7}$ and to a diphoton decay rate in two gluons $\Gamma_{g g} / M_{\digamma} \simeq 4.7 \times 10^{-5}$. This example employs $N=12$, which is compatible with experimental bounds.

Given that such a large $N$ might look implausible from a low-energy perspective, we provide in the lower row a second example with $N=5$, achieved by increasing $Y=4 / 3$. This example has $M_{X}=1.6 \mathrm{TeV}, \Gamma_{\gamma \gamma} \simeq 4.4 \times 10^{-7} M_{\digamma}, \Gamma_{g g} \simeq 4.2 \times 10^{-6} M_{\digamma}$.

In both cases the $\mathrm{SU}(N)$ gauge constant becomes relatively large at the diphoton scale such that the couplings (in particular $\lambda_{X S}$ ) have a fast running; we tried to include such corrections by renormalising couplings at appropriate scales.

\section{Strongly coupled models}

In this section we try to build dimensionless models where a new gauge interaction (we use for it the old-fashioned name TechniColor, or TC) becomes strong around the weak scale, inducing the weak scale and the diphoton $\digamma$. Like in the weakly-coupled case, it is easy to reproduce the diphoton excess by adding one extra ad-hoc diphoton scalar (see [43] for one 

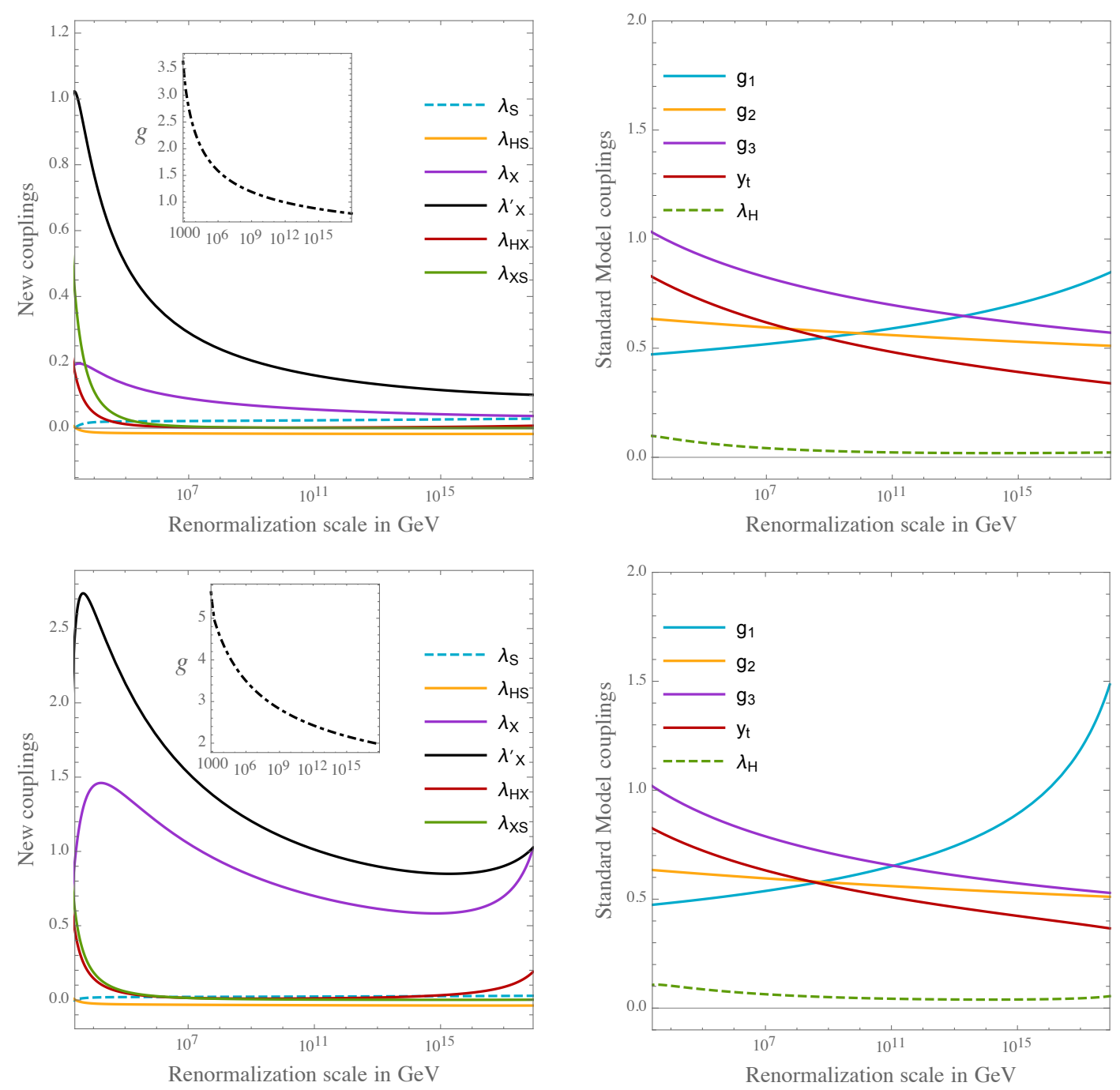

Figure 3. Running of the couplings for central values for the SM parameters, that we took from [80], and $g^{2}\left(M_{\digamma}\right)=(4 \pi)^{2} / N$. We find that that varying the values of $\lambda_{X}\left(M_{\digamma}\right), \lambda_{X}^{\prime}\left(M_{\digamma}\right), \lambda_{H X}\left(M_{\digamma}\right)$ have a very small impact on the running at energies much bigger than $\digamma_{*}$. Upper plots: we assumed $Y=2 / 3, N=12, w=1890 \mathrm{GeV}, 5 N$ fermions in the $N \oplus \bar{N}$ representation of $\operatorname{SU}(N)$; we also take $\lambda_{X}\left(M_{\digamma}\right)=0.15, \lambda_{X}^{\prime}\left(M_{\digamma}\right)=0.28, \lambda_{H X}\left(M_{\digamma}\right)=0.17$. Lower plots: we assumed $Y=4 / 3, N=5$, $w=2000 \mathrm{GeV}, 26$ fermions in the $N \oplus \bar{N}$ representation of $\mathrm{SU}(N)$; we also take $\lambda_{X}\left(M_{\digamma}\right)=0.15$, $\lambda_{X}^{\prime}\left(M_{\digamma}\right)=0.28, \lambda_{H X}\left(M_{\digamma}\right)=0.47$.

such model). We are interested in models where the diphoton automatically emerges as a bound state of the TC dynamics. In view of strong LHC bounds on extra bound states, especially the ones with color, plausible models identify the diphoton with a bound state that is much lighter than the others. The diphoton could be a $\mathrm{TC} \eta$ (here discussed in section 3.1) or a TC-dilaton (here discussed in section 3.2). The TC-dilaton is especially interesting from our point of view, given that it is the pseudo-Goldstone boson of scale invariance. 


\begin{tabular}{|cc|cccc|}
\hline name & spin & $\mathrm{SU}(3)_{c}$ & $\mathrm{SU}(2)_{L}$ & $\mathrm{U}(1)_{Y}$ & $\mathrm{SU}(N)$ \\
\hline $\mathcal{Q}$ & $1 / 2$ & 3 & 2 & $+1 / 6$ & $N$ \\
$\mathcal{Q}^{c}$ & $1 / 2$ & $\overline{3}$ & 2 & $-1 / 6$ & $\bar{N}$ \\
$\mathcal{U}$ & $1 / 2$ & $\overline{3}$ & 1 & $-2 / 3$ & $N$ \\
$\mathcal{U}^{c}$ & $1 / 2$ & 3 & 1 & $+2 / 3$ & $\bar{N}$ \\
\hline
\end{tabular}

Table 3. Beyond the Standard Model field content of the strongly-coupled model of section 3.1.

\subsection{The diphoton as a TC $\eta$}

A class of strongly-coupled models that aims at reproducing the diphoton excess are those where the diphoton $\digamma$ is identified with a TC $\eta$ composite pseudo-scalar, given that this field is a light pseudo-Goldstone boson with anomalous couplings to SM vectors. The various models proposed in the literature [46, 68, 70, 92-100] employ massive techni-quarks, and a massive SM Higgs doublet. The masses are assumed to be comparable to the TC scale, but the coincidence is left unexplained.

Our goal is exploring whether such masses can be all set to zero, obtaining a dimensionless model. For concreteness, we consider a model with $G_{\mathrm{TC}}=\mathrm{SU}(N)$ and the techni-quark content of table $3^{4}$ which allows for two Yukawa couplings to the SM Higgs doublet $H$ :

$$
y_{1} H \mathcal{Q U}+y_{2} H^{\dagger} \mathcal{Q}^{c} \mathcal{U}^{c}+\text { h.c. }=H \bar{\Psi}_{\mathcal{Q}}\left(y+i \gamma_{5} \tilde{y}\right) \Psi_{\mathcal{U}}+\text { h.c. }
$$

In the latter expression we introduced Dirac spinors $\Psi$ and the scalar coupling $y=\left(y_{1}+\right.$ $\left.y_{2}^{*}\right) / 2$ and the pseudo-scalar coupling $\tilde{y}=i\left(y_{1}-y_{2}^{*}\right) / 2$, such that $|y| \ll|\tilde{y}|$ and $|\tilde{y}| \ll|y|$ are radiatively stable special cases, that can be justified by assuming a CP-like symmetry.

We now discuss the composite states. Among the many states around the TC $\rho$ mass, $m_{\rho} \sim g_{\rho} f_{\mathrm{TC}}$ with $g_{\rho} \sim 4 \pi / \sqrt{N}$, there is the TC $\eta^{\prime} \sim Q Q^{c}+U U^{c}$ singlet which receives a mass from TC anomalies. The TC dynamics breaks the global accidental symmetry $\mathrm{SU}(9)_{L} \otimes \mathrm{SU}(9)_{R} \rightarrow \mathrm{SU}(9)_{V}$ giving 80 lighter techni-pions, with the following SM quantum numbers:

$$
(1,1)_{0} \oplus(1,2)_{ \pm 1 / 2} \oplus(1,3)_{0} \oplus 2(8,1)_{0} \oplus(8,2)_{ \pm 1 / 2} \oplus(8,3)_{0}
$$

Taking into account that we assume massless techni-quarks, the techni-pions consist of:

72) 9 color octets, $Q Q^{c}, U U^{c}, Q U^{c}, Q^{c} U$, which get positive squared masses at loop level from QCD interactions, $m \approx \sqrt{\frac{3}{4 \pi} \alpha_{3} C} m_{\rho}^{2} \sim 0.2 m_{\rho}$, where $C=\left(N^{2}-1\right) / 2 N$;

3) a $\mathrm{SU}(2)_{L}$ triplet $Q Q^{c}$, which similarly gets a positive squared mass from weak interactions;

1) a TC $\eta \sim U U^{c}-\frac{1}{2} Q Q^{c}$ singlet (to be identified with the diphoton), which gets a squared mass from Yukawa loop corrections with unknown sign and of order $m_{\eta} \sim$ $y m_{\rho} / 4 \pi$, see appendix A.3 of [39];

\footnotetext{
${ }^{4}$ A similar model with up-type quarks replaced by down-type quarks has been considered in [101] because the lightest TCbaryon is a good Dark Matter candidate.
} 

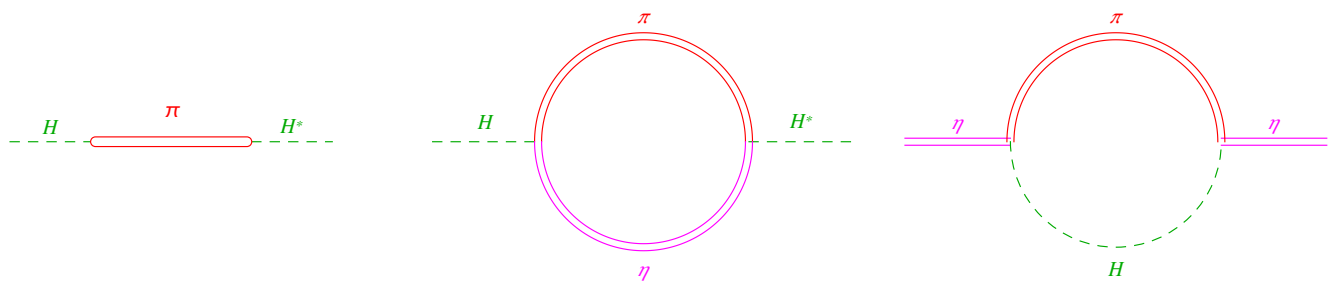

Figure 4. Correction to the Higgs and diphoton mass coming from the Yukawa couplings.

4) a TCpion $\pi_{2}$ from $Q U^{c}, Q^{c} U$ with the same gauge quantum numbers as the Higgs doublet forms whenever gauge quantum numbers allow for a Yukawa coupling to $H$. It gets a mass $m_{\pi_{2}} \sim g_{2} m_{\rho} / 4 \pi$ from weak gauge interactions, plus another contribution from $y_{1,2}$.

Furthermore, $\pi_{2}$ acquires a tree level mixing with the Higgs boson. The resulting mass matrix is

$$
\begin{aligned}
& \pi_{2}^{*} \quad H^{*} \\
& \begin{array}{l}
\pi_{2} \\
H
\end{array}\left(\begin{array}{cc}
\left(\mathcal{O}\left(g_{2}^{2}\right) \pm \mathcal{O}\left(y^{2}\right)\right) /(4 \pi)^{2} & \mathcal{O}(y) \sqrt{N} /(4 \pi) \\
\mathcal{O}(y) \sqrt{N} /(4 \pi) & -\mathcal{O}\left(y^{2}\right) N /(4 \pi)^{2}
\end{array}\right) m_{\rho}^{2}
\end{aligned}
$$

The tree-level mixing induces a negative see-saw contribution $\Delta m_{H}^{2} \sim-\tilde{y}^{2} m_{\rho}^{2} f_{\mathrm{TC}}^{2} / m_{\pi_{2}}^{2}$ to the Higgs mass parameter. This contribution is too big, given that the diphoton only gets a mass at loop level.

Such tree-level contribution vanishes if we assume $\tilde{y}=0-$ a natural special case that respects a CP-like parity. Indeed, $H$ is a scalar, while $\pi_{2}$ and $\eta$ are pseudo-scalars. Their tree-level potential includes a CP-conserving term $\sim y m_{\rho} H \pi_{2}^{*} \eta+$ h.c. As a result, both $H$ and of $\eta$ receive loop-level masses of order $y m_{\rho} / 4 \pi$, with no symmetry relation among them, given that $H$ is elementary while $\eta$ is composite, see figure 4 . With a relatively large value of $y \sim$ few the model can give $M_{\digamma} \approx 750 \mathrm{GeV}$ together with $f_{\mathrm{TC}} \approx 100 \mathrm{GeV} \times N$ as demanded by the diphoton rate suggested by preliminary ATLAS and CMS results. The $\eta$ eigenstate has a vanishing color anomaly (see table 2 of [98]); this is not a problem because, taking into loop corrections, the mass eigenstate $\digamma \approx \eta+\eta^{\prime} y^{2} /(4 \pi)^{2}$ acquires a sufficiently large coupling to gluons.

\subsection{The diphoton as a TC-dilaton or TC $\sigma$}

In strongly coupled TC models with a QCD-like dynamics the mass of the pseudo-Goldstone boson of scale invariance is not suppressed with respect to the other composite states, because strong interactions give a fast running that strongly breaks scale invariance. On the other hand, such state is somehow lighter than the other bound states in models with a 'walking' dynamics (namely, a $\beta$ function of the new gauge interaction which remains somewhat small), which is obtained in TC models with a larger matter content than QCD. This lightness is beneficial for the diphoton phenomenology, given that other colored bound states must be heavier than $750 \mathrm{GeV}$ in order to satisfy LHC bounds. 
From a low-energy perspective, such light state is the $\sigma$ field sometimes explicitly included in effective chiral Lagrangians, where its vacuum expectation value, $\sigma=f_{\mathrm{TC}}+\digamma$, breaks scale invariance as well as a global chiral symmetry, such that $f_{\mathrm{TC}}$ becomes the techni-pion decay constant. See [102] for a recent discussion of the $\sigma$, and [46, 103-105] for recent discussions of the dilaton. In the limit where $\digamma$ is lighter than the other bound states, its coupling to SM vectors is dictated by eq. (2.12): using $\ln M_{\wp}(\digamma) / M_{\wp} \simeq \digamma / f_{\mathrm{TC}}$ we find

$$
\frac{\Gamma_{\gamma \gamma}}{M_{\digamma}}=10^{-6}\left(\frac{120 \mathrm{GeV}}{f_{\mathrm{TC}}}\right)^{2} \Delta b_{\mathrm{em}}^{2}
$$

where $\Delta b_{\text {em }}$ is the techniquark contribution to the running of the electromagnetic coupling. For example, a color triplet techni-fermion with hypercharge $Y$ (such as $\mathcal{U} \oplus \mathcal{U}^{c}$ in table 1) contributes as $\Delta b_{\mathrm{em}}=4 N Y^{2}$, while a techni-scalar contributes 4 times less. The SM $\Delta b_{Y, 2,3}$ must be smaller than about 10, in order to avoid sub-Planckian Landau poles for the SM gauge couplings.

The dynamically generated Higgs mass depends on the model. In the minimal case where the Higgs has no direct coupling to techni-particles, electro-weak loop effects induce a contribution to its squared mass of order $M_{h}^{2} \sim-\alpha_{Y}^{2} f_{\text {TC }}^{2}$ [39], which is negative but small, in view of eq. (3.4). A larger model-dependent tree-level contribution is obtained if the Higgs has a Yukawa coupling to techni-fermions or a quartic coupling to techni-scalars.

\section{Conclusions}

We proposed dimension-less models where a new resonance $\digamma$ decaying into two photons dynamically breaks scale invariance, generating a weak scale hierarchically smaller than the Planck scale. The diphoton channel is interesting as it is particularly clean and has therefore a great discovery potential. All particles acquire their masses from their couplings to $\digamma$ : thereby the smoking gun of this scenario is observing that $\digamma$ couples to all particles proportionally to their mass.

As a benchmark case we identified $\digamma$ with the $750 \mathrm{GeV}$ resonance hinted by LHC data. Although it could very well be that this excess is a statistical fluctuation, it nevertheless provides an interesting example. The $\digamma \rightarrow \gamma \gamma$ rate suggested by LHC data is obtained adding extra charged particles, heavier than the SM particles and thereby more strongly coupled to $\digamma$.

Diphoton models of this type generically need that such extra particles have masses around the weak scale. Unlike the SM fermions, such extra particles have no chirality reason to be around the weak scale. Scale invariance provides one possible reason: like the Higgs, these extra charged particles acquire a mass from $\digamma$. All particles are massless until the diphoton develops a vev or condensate $w$.

A generic scalar that acts as the 'Higgs of the Higgs' can have a $w$ and/or a mass $M$ much larger than the weak scale, provided that it is very weakly coupled to the Standard Model (SM). This is not possible if such scalar is identified with the diphoton: $w$ and $M$ are now fixed by the diphoton mass and rate in $\gamma \gamma$. 
In section 2.1 we presented a weakly-coupled dimension-less model where the diphoton is charged under extra gauge interactions, which induce the Coleman-Weinberg mechanism. In section 2.2 we presented a weakly-coupled dimension-less model where the ColemanWeinberg mechanism is induced by quartic interactions of the diphoton with the charged scalars that mediate $\digamma \rightarrow \gamma \gamma$.

Both models can be RGE-extrapolated up to the Planck scale, thereby generating the large hierarchy with respect to the weak scale. Both models contain Dark Matter candidates. Both models remove the instability of the SM potential. Both models give rise to an extended phase transition when the diphoton and the Higgs acquire vacuum expectation values. Such phase transition can be of first order, possibly giving gravitational wave signals [106] and the baryon asymmetry [107].

In section 3.1 we discussed dimension-less strongly-coupled models where the diphoton is a pseudo-scalar bound state analogous of the $\eta$ in QCD. In section 3.2 we discussed dimension-less strongly-coupled models where the diphoton is a scalar bound state analogous of the $\sigma$ in chiral effective Lagrangians, or of a dirty dilaton in fundamental strongly coupled models with walking dynamics. Both scenarios can produce a weak scale lighter than the diphoton mass by $M_{h} / M_{\digamma} \sim 1 / 6$, but this needs extra model building features.

\section{Acknowledgments}

This work was supported by the grant 669668 - NEO-NAT - ERC-AdG-2014. Kristjan Kannike was supported by the Estonian Research Council grant PUT799, the grant IUT236 of the Estonian Ministry of Education and Research, and by the EU through the ERDF CoE program. We thank Roberto Franceschini and Francesco Riva for useful discussions.

\section{A RGE for model $g$ )}

Defining $\beta_{g}=d g / d \ln \mu$, the one-loop RGE for the new couplings are

$$
\begin{aligned}
(4 \pi)^{2} \beta_{\lambda_{S}}= & 4(4+N) \lambda_{S}^{2}+\frac{3(N-1)\left(N^{2}+2 N-2\right)}{4 N^{2}} g^{4} \\
& +2\left(\lambda_{S X}^{2}+\lambda_{H S}^{2}\right)-6 y_{S}^{4}+\lambda_{S}\left[12 y_{S}^{2}-6 \frac{N^{2}-1}{N} g^{2}\right] \\
\left(4 \pi^{2}\right) \beta_{\lambda_{H S}}= & 12 y_{S}^{2} y_{t}^{2}-4 \lambda_{H S}^{2}-4 \lambda_{H X} \lambda_{S X} \\
& +\lambda_{H S}\left[-3 g^{2} \frac{N^{2}-1}{N}-\frac{9 g_{1}^{2}}{10}-\frac{9 g_{2}^{2}}{2}+6 y_{S}^{2}+6 y_{t}^{2}+12 \lambda_{H}+(4 N+4) \lambda_{S}\right] \\
\left(4 \pi^{2}\right) \beta_{\lambda_{H X}}= & -8 \lambda_{H X}^{2}-2 N \lambda_{H S} \lambda_{S X} \\
& +\lambda_{H X}\left[-\frac{9 g_{1}^{2}}{10}-\frac{9 g_{2}^{2}}{2}+6 y_{t}^{2}+12 N y_{X}^{2}+12 \lambda_{H}+24 \lambda_{X}\right] \\
\left(4 \pi^{2}\right) \beta_{\lambda_{S X}}= & 12 y_{S}^{2} y_{X}^{2}-8 \lambda_{S X}^{2}-4 \lambda_{H S} \lambda_{H X} \\
& +\lambda_{S X}\left[-3 g^{2} \frac{N^{2}-1}{N}+6 y_{S}^{2}+12 N y_{X}^{2}+(4 N+4) \lambda_{S}+24 \lambda_{X}\right]
\end{aligned}
$$




$$
\begin{aligned}
\left(4 \pi^{2}\right) \beta_{\lambda_{X}} & =-6 N y_{X}^{4}+24 N \lambda_{X} y_{X}^{2}+2 \lambda_{H X}^{2}+N \lambda_{S X}^{2}+72 \lambda_{X}^{2} \\
\left(4 \pi^{2}\right) \beta_{y_{X}} & =(6 N+3) y_{X}^{3}+y_{X}\left[-3 \frac{N^{2}-1}{N} g^{2}-\frac{8 g_{1}^{2}}{5}-8 g_{3}^{2}+\frac{y_{S}^{2}}{2}\right] \\
\left(4 \pi^{2}\right) \beta_{y_{S}} & =\frac{N+7}{2} y_{S}^{3}+y_{S}\left[-\frac{3}{2} g^{2} \frac{N^{2}-1}{N}-\frac{8 g_{1}^{2}}{5}-8 g_{3}^{2}+y_{t}^{2}+\frac{y_{X}^{2}}{2}\right] \\
\left(4 \pi^{2}\right) \beta_{g} & =\left(\frac{13}{6}-\frac{11 N}{3}\right) g^{3} .
\end{aligned}
$$

Finally, the RGE for the SM couplings are

$$
\begin{aligned}
\left(4 \pi^{2}\right) \beta_{g_{1}}= & \left(\frac{41}{10}+\Delta b_{1}\right) g_{1}^{3}, \quad \Delta b_{1}=\frac{16 N}{15} \\
\left(4 \pi^{2}\right) \beta_{g_{2}}= & -\frac{19 g_{2}^{3}}{6}, \\
\left(4 \pi^{2}\right) \beta_{g_{3}}= & \left(-7+\Delta b_{3}\right) g_{3}^{3}, \quad \Delta b_{3}=\frac{2 N}{3}, \\
\left(4 \pi^{2}\right) \beta_{y_{t}}= & \frac{9 y_{t}^{3}}{2}+y_{t}\left[-\frac{17 g_{1}^{2}}{20}-\frac{9 g_{2}^{2}}{4}-8 g_{3}^{2}+\frac{N y_{S}^{2}}{2}\right] \\
\left(4 \pi^{2}\right) \beta_{\lambda_{H}}= & \frac{27 g_{1}^{4}}{200}+\frac{9 g_{2}^{2} g_{1}^{2}}{20}+\frac{9 g_{2}^{4}}{8}-6 y_{t}^{4}+24 \lambda_{H}^{2}+N \lambda_{H S}^{2}+2 \lambda_{H X}^{2} \\
& +\lambda_{H}\left[12 y_{t}^{2}-\frac{9 g_{1}^{2}}{5}-9 g_{2}^{2}\right] .
\end{aligned}
$$

\section{B RGE for model $\lambda$ )}

Defining $\beta_{g}=d g / d \ln \mu$, the RGE for the new couplings are

$$
\begin{aligned}
(4 \pi)^{2} \beta_{\lambda_{S}}= & 72 \lambda_{S}^{2}+3 N \lambda_{X S}^{2}+2 \lambda_{H S}^{2} \\
(4 \pi)^{2} \beta_{\lambda_{X}}= & 4(3 N+4) \lambda_{X}^{2}+12 \lambda_{X}^{\prime 2}+2 \lambda_{X S}^{2}+2 \lambda_{H X}^{2} \\
& +\lambda_{X}\left[8(3+N) \lambda_{X}^{\prime}-\frac{6\left(N^{2}-1\right)}{N} g^{2}-16 g_{3}^{2}-\frac{36 Y^{2} g_{1}^{2}}{5}+\frac{54 Y^{4}}{25} g_{1}^{4}\right] \\
& +\frac{3\left(N^{2}+2\right)}{4 N^{2}} g^{4}+\frac{11}{12} g_{3}^{4}+\frac{3 N+1}{N} g^{2} g_{3}^{2}-\frac{18 Y^{2} g^{2} g_{1}^{2}}{5 N}-\frac{6 Y^{2} g_{3}^{2} g_{1}^{2}}{5} \\
(4 \pi)^{2} \beta_{\lambda_{X}^{\prime}}= & 4(3+N) \lambda_{X}^{\prime 2}+\lambda_{X}^{\prime}\left[24 \lambda_{X}-\frac{36 Y^{2} g_{1}^{2}}{5}-\frac{6\left(N^{2}-1\right)}{N} g^{2}-16 g_{3}^{2}\right] \\
& +\frac{3\left(N^{2}-4\right)}{4 N} g^{4}+\frac{5 g_{3}^{4}}{4}-\frac{N+3}{N} g^{2} g_{3}^{2}+\frac{18 Y^{2} g^{2} g_{1}^{2}}{5}+\frac{18 Y^{2} g_{3}^{2} g_{1}^{2}}{5} \\
(4 \pi)^{2} \beta_{\lambda_{X S}}= & 8 \lambda_{X S}^{2}-4 \lambda_{H S} \lambda_{H X}+4 \lambda_{X S}\left[(1+3 N) \lambda_{X}+(3+N) \lambda_{X}^{\prime}\right. \\
& \left.+6 \lambda_{S}-\frac{9 Y^{2} g_{1}^{2}}{10}-\frac{3\left(N^{2}-1\right)}{4 N} g^{2}-2 g_{3}^{2}\right], \\
& \lambda_{H X}\left[4(1+3 N) \lambda_{X}+4(3+N) \lambda_{X}^{\prime}-\frac{\left(36 Y^{2}+9\right) g_{1}^{2}}{10}-\frac{9 g_{2}^{2}}{2}+12 \lambda_{H}+6 y_{t}^{2}\right] \\
& -4 \lambda_{H S} \lambda_{X S}+4 \lambda_{H X}^{2}+\frac{27 g_{1}^{4} Y^{2}}{25}-\frac{3\left(N^{2}-1\right)}{N} \lambda_{H X} g^{2}-8 \lambda_{H X} g_{3}^{2}
\end{aligned}
$$




$$
\begin{aligned}
(4 \pi)^{2} \beta_{\lambda_{H S}} & =\lambda_{H S}\left[24 \lambda_{S}-\frac{9 g_{1}^{2}}{10}-\frac{9 g_{2}^{2}}{2}+6 y_{t}^{2}+12 \lambda_{H}\right]-6 N \lambda_{X S} \lambda_{H X}-8 \lambda_{H S}^{2}, \\
(4 \pi)^{2} \beta_{g} & =g^{3}\left(-\frac{11}{3} N+\frac{1}{2}+\frac{2}{3} N_{f}\right) .
\end{aligned}
$$

Finally, the RGE for the SM couplings are

$$
\begin{aligned}
(4 \pi)^{2} \beta_{g_{1}}= & g_{1}^{3} \frac{41+6 N Y^{2}}{10} \\
(4 \pi)^{2} \beta_{g_{2}}= & -\frac{19 g_{2}^{3}}{6} \\
(4 \pi)^{2} \beta_{g_{3}}= & g_{3}^{3}\left(-7+\frac{N}{6}\right) \\
(4 \pi)^{2} \beta_{\lambda_{H}}= & 2 \lambda_{H S}^{2}+3 N \lambda_{H X}^{2}+\frac{27 g_{1}^{4}}{200}+\frac{9 g_{1}^{2} g_{2}^{2}}{20}+\frac{9 g_{2}^{4}}{8} \\
& +\lambda_{H}\left(-\frac{9 g_{1}^{2}}{5}-9 g_{2}^{2}+12 y_{t}^{2}\right)+24 \lambda_{H}^{2}-6 y_{t}^{4} \\
(4 \pi)^{2} \beta_{y_{t}}= & y_{t}\left(\frac{9}{2} y_{t}^{2}-\frac{17 g_{1}^{2}}{20}-8 g_{3}^{2}-\frac{9 g_{2}^{2}}{4}\right) .
\end{aligned}
$$

Open Access. This article is distributed under the terms of the Creative Commons Attribution License (CC-BY 4.0), which permits any use, distribution and reproduction in any medium, provided the original author(s) and source are credited.

\section{References}

[1] M. Farina, D. Pappadopulo and A. Strumia, A modified naturalness principle and its experimental tests, JHEP 08 (2013) 022 [arXiv: 1303.7244] [INSPIRE].

[2] M. Heikinheimo, A. Racioppi, M. Raidal, C. Spethmann and K. Tuominen, Physical naturalness and dynamical breaking of classical scale invariance, Mod. Phys. Lett. A 29 (2014) 1450077 [arXiv:1304.7006] [INSPIRE].

[3] S.L. Adler, A formula for the induced gravitational constant, Phys. Lett. B 95 (1980) 241 [INSPIRE].

[4] A. Zee, Spontaneously generated gravity, Phys. Rev. D 23 (1981) 858 [InSPIRE].

[5] A. Salvio and A. Strumia, Agravity, JHEP 06 (2014) 080 [arXiv: 1403.4226] [INSPIRE].

[6] M. Rinaldi, G. Cognola, L. Vanzo and S. Zerbini, Inflation in scale-invariant theories of gravity, Phys. Rev. D 91 (2015) 123527 [arXiv:1410.0631] [INSPIRE].

[7] K. Kannike et al., Dynamically induced Planck scale and inflation, JHEP 05 (2015) 065 [arXiv: 1502.01334] [INSPIRE].

[8] A. Farzinnia and S. Kouwn, Classically scale invariant inflation, supermassive WIMPs and adimensional gravity, Phys. Rev. D 93 (2016) 063528 [arXiv:1512.05890] [INSPIRE].

[9] A. Farzinnia, Classically scale invariant inflation and (A)gravity, Int. J. Mod. Phys. Conf. Ser. 43 (2016) 1660203 [arXiv: 1512.08727] [INSPIRE]. 
[10] A. Salvio and A. Strumia, Quantum mechanics of 4-derivative theories, Eur. Phys. J. C 76 (2016) 227 [arXiv: 1512.01237] [inSPIRE].

[11] M.B. Einhorn and D.R.T. Jones, Induced gravity II: grand unification, JHEP 05 (2016) 185 [arXiv: 1602.06290] [INSPIRE].

[12] T. Hambye and A. Strumia, Dynamical generation of the weak and dark matter scale, Phys. Rev. D 88 (2013) 055022 [arXiv: 1306.2329] [INSPIRE].

[13] R. Foot, A. Kobakhidze, K. McDonald and R. Volkas, Neutrino mass in radiatively-broken scale-invariant models, Phys. Rev. D 76 (2007) 075014 [arXiv:0706.1829] [InSPIRE].

[14] R. Foot, A. Kobakhidze, K.L. McDonald and R.R. Volkas, A solution to the hierarchy problem from an almost decoupled hidden sector within a classically scale invariant theory, Phys. Rev. D 77 (2008) 035006 [arXiv:0709.2750] [INSPIRE].

[15] R. Foot, A. Kobakhidze and R.R. Volkas, Stable mass hierarchies and dark matter from hidden sectors in the scale-invariant standard model, Phys. Rev. D 82 (2010) 035005 [arXiv: 1006.0131] [INSPIRE].

[16] R. Foot and A. Kobakhidze, Electroweak scale invariant models with small cosmological constant, Int. J. Mod. Phys. A 30 (2015) 1550126 [arXiv:1112.0607] [INSPIRE].

[17] L. Alexander-Nunneley and A. Pilaftsis, The minimal scale invariant extension of the standard model, JHEP 09 (2010) 021 [arXiv: 1006.5916] [INSPIRE].

[18] K. Ishiwata, Dark matter in classically scale-invariant two singlets standard model, Phys. Lett. B 710 (2012) 134 [arXiv:1112.2696] [INSPIRE].

[19] J.S. Lee and A. Pilaftsis, Radiative corrections to scalar masses and mixing in a scale invariant two Higgs doublet model, Phys. Rev. D 86 (2012) 035004 [arXiv:1201.4891] [INSPIRE].

[20] A. Farzinnia, H.-J. He and J. Ren, Natural electroweak symmetry breaking from scale invariant Higgs mechanism, Phys. Lett. B 727 (2013) 141 [arXiv: 1308.0295] [INSPIRE].

[21] D. Chway, T.H. Jung, H.D. Kim and R. Dermisek, Radiative electroweak symmetry breaking model perturbative all the way to the Planck scale, Phys. Rev. Lett. 113 (2014) 051801 [arXiv:1308.0891] [INSPIRE].

[22] E. Gabrielli et al., Towards completing the standard model: vacuum stability, EWSB and dark matter, Phys. Rev. D 89 (2014) 015017 [arXiv: 1309.6632] [InSPIRE].

[23] C.T. Hill, Is the Higgs Boson Associated with Coleman-Weinberg Dynamical Symmetry Breaking?, Phys. Rev. D 89 (2014) 073003 [arXiv:1401.4185] [INSPIRE].

[24] J. Guo and Z. Kang, Higgs naturalness and dark matter stability by scale invariance, Nucl. Phys. B 898 (2015) 415 [arXiv: 1401.5609] [InSPIRE].

[25] H. Davoudiasl and I.M. Lewis, Right-handed neutrinos as the origin of the electroweak scale, Phys. Rev. D 90 (2014) 033003 [arXiv:1404.6260] [InSPIRE].

[26] K. Allison, C.T. Hill and G.G. Ross, Ultra-weak sector, Higgs boson mass and the dilaton, Phys. Lett. B 738 (2014) 191 [arXiv: 1404.6268] [INSPIRE].

[27] A. Farzinnia and J. Ren, Higgs partner searches and dark matter phenomenology in a classically scale invariant Higgs boson sector, Phys. Rev. D 90 (2014) 015019 [arXiv: 1405.0498] [INSPIRE]. 
[28] M. Lindner, S. Schmidt and J. Smirnov, Neutrino masses and conformal electro-weak symmetry breaking, JHEP 10 (2014) 177 [arXiv:1405.6204] [INSPIRE].

[29] K. Allison, C.T. Hill and G.G. Ross, An ultra-weak sector, the strong CP problem and the pseudo-Goldstone dilaton, Nucl. Phys. B 891 (2015) 613 [arXiv:1409.4029] [InSPIRE].

[30] K. Endo and Y. Sumino, A scale-invariant Higgs sector and structure of the vacuum, JHEP 05 (2015) 030 [arXiv: 1503.02819] [inSPIRE].

[31] A. Karam and K. Tamvakis, Dark matter and neutrino masses from a scale-invariant multi-Higgs portal, Phys. Rev. D 92 (2015) 075010 [arXiv:1508.03031] [INSPIRE].

[32] A. Ahriche, K.L. McDonald and S. Nasri, The scale-invariant scotogenic model, JHEP 06 (2016) 182 [arXiv: 1604.05569] [inSPIRE].

[33] S. Benic and B. Radovcic, Electroweak breaking and dark matter from the common scale, Phys. Lett. B 732 (2014) 91 [arXiv:1401.8183] [INSPIRE].

[34] G.M. Pelaggi, Predictions of a model of weak scale from dynamical breaking of scale invariance, Nucl. Phys. B 893 (2015) 443 [arXiv:1406.4104] [INSPIRE].

[35] W. Altmannshofer, W.A. Bardeen, M. Bauer, M. Carena and J.D. Lykken, Light dark matter, naturalness and the radiative origin of the electroweak scale, JHEP 01 (2015) 032 [arXiv: 1408.3429] [INSPIRE].

[36] A.D. Plascencia, Classical scale invariance in the inert doublet model, JHEP 09 (2015) 026 [arXiv: 1507.04996] [INSPIRE].

[37] N. Haba, H. Ishida, N. Okada and Y. Yamaguchi, Bosonic seesaw mechanism in a classically conformal extension of the Standard Model, Phys. Lett. B 754 (2016) 349 [arXiv: 1508.06828] [INSPIRE].

[38] A. Karam and K. Tamvakis, Dark matter and neutrino masses from a classically scale-invariant multi-Higgs portal, arXiv:1603.08470 [INSPIRE].

[39] O. Antipin, M. Redi and A. Strumia, Dynamical generation of the weak and Dark Matter scales from strong interactions, JHEP 01 (2015) 157 [arXiv:1410.1817] [INSPIRE].

[40] M. Holthausen, J. Kubo, K.S. Lim and M. Lindner, Electroweak and conformal symmetry breaking by a strongly coupled hidden sector, JHEP 12 (2013) 076 [arXiv:1310.4423] [INSPIRE].

[41] J. Kubo, K.S. Lim and M. Lindner, Electroweak symmetry breaking via QCD, Phys. Rev. Lett. 113 (2014) 091604 [arXiv: 1403.4262] [INSPIRE].

[42] C.D. Carone and R. Ramos, Dark chiral symmetry breaking and the origin of the electroweak scale, Phys. Lett. B 746 (2015) 424 [arXiv: 1505.04448] [INSPIRE].

[43] H. Ishida, S. Matsuzaki and Y. Yamaguchi, Invisible axion-like dark matter from electroweak bosonic seesaw, arXiv:1604.07712 [INSPIRE].

[44] ATLAS collaboration, Search for resonances decaying to photon pairs in $3.2 \mathrm{fb}^{-1}$ of $\mathrm{pp}$ collisions at $\sqrt{s}=13 \mathrm{TeV}$ with the ATLAS detector, ATLAS-CONF-2015-081 (2015).

[45] CMS collaboration, Search for new physics in high mass diphoton events in proton-proton collisions at $13 \mathrm{TeV}$, CMS-PAS-EXO-15-004 (2015).

[46] R. Franceschini et al., What is the $\gamma \gamma$ resonance at 750 GeV?, JHEP 03 (2016) 144 [arXiv: 1512.04933] [INSPIRE]. 
[47] S. Di Chiara, L. Marzola and M. Raidal, First interpretation of the $750 \mathrm{GeV}$ diphoton resonance at the LHC, Phys. Rev. D 93 (2016) 095018 [arXiv: 1512.04939] [InSPIRE].

[48] C. Petersson and R. Torre, $750 \mathrm{GeV}$ diphoton excess from the goldstino superpartner, Phys. Rev. Lett. 116 (2016) 151804 [arXiv:1512.05333] [INSPIRE].

[49] S.V. Demidov and D.S. Gorbunov, On the sgoldstino interpretation of the diphoton excess, JETP Lett. 103 (2016) 219 [arXiv:1512.05723] [INSPIRE].

[50] T.-F. Feng, X.-Q. Li, H.-B. Zhang and S.-M. Zhao, The LHC $750 \mathrm{GeV}$ diphoton excess in supersymmetry with gauged baryon and lepton numbers, arXiv:1512.06696 [INSPIRE].

[51] L.M. Carpenter, R. Colburn and J. Goodman, Supersoft SUSY models and the $750 \mathrm{GeV}$ diphoton excess, beyond effective operators, Phys. Rev. D 94 (2016) 015016 [arXiv: 1512.06107] [INSPIRE].

[52] R. Ding, L. Huang, T. Li and B. Zhu, Interpreting $750 \mathrm{GeV}$ diphoton excess with R-parity violating supersymmetry, arXiv:1512.06560 [INSPIRE].

[53] F. Wang, L. Wu, J.M. Yang and M. Zhang, $750 \mathrm{GeV}$ diphoton resonance, $125 \mathrm{GeV}$ Higgs and muon $g-2$ anomaly in deflected anomaly mediation SUSY breaking scenarios, Phys. Lett. B 759 (2016) 191 [arXiv: 1512.06715] [INSPIRE].

[54] B.C. Allanach, P.S.B. Dev, S.A. Renner and K. Sakurai, $750 \mathrm{GeV}$ diphoton excess explained by a resonant sneutrino in R-parity violating supersymmetry, Phys. Rev. D 93 (2016) 115022 [arXiv: 1512.07645] [INSPIRE].

[55] J.A. Casas, J.R. Espinosa and J.M. Moreno, The $750 \mathrm{GeV}$ diphoton excess as a first light on supersymmetry breaking, Phys. Lett. B 759 (2016) 159 [arXiv:1512.07895] [INSPIRE].

[56] L.J. Hall, K. Harigaya and Y. Nomura, $750 \mathrm{GeV}$ diphotons: implications for supersymmetric unification, JHEP 03 (2016) 017 [arXiv: 1512.07904] [INSPIRE].

[57] Y. Jiang, Y.-Y. Li and T. Liu, $750 \mathrm{GeV}$ resonance in the gauged $\mathrm{U}(1)^{\prime}$-extended MSSM, Phys. Lett. B 759 (2016) 354 [arXiv:1512.09127] [INSPIRE].

[58] W. Chao, The diphoton excess from an exceptional supersymmetric standard model, arXiv: 1601.00633 [INSPIRE].

[59] B. Dutta et al., Diphoton excess in consistent supersymmetric SU(5) models with vector-like particles, arXiv:1601.00866 [INSPIRE].

[60] S.F. King and R. Nevzorov, $750 \mathrm{GeV}$ diphoton resonance from singlets in an exceptional supersymmetric standard model, JHEP 03 (2016) 139 [arXiv:1601.07242] [INSPIRE].

[61] G. Lazarides and Q. Shafi, Diphoton resonances in a $\mathrm{U}(1)_{B-L}$ extension of the minimal supersymmetric standard model, Phys. Rev. D 93 (2016) 111702 [arXiv:1602.07866] [INSPIRE].

[62] R. Ding et al., Systematic study of diphoton resonance at $750 \mathrm{GeV}$ from sgoldstino, arXiv: 1602.00977 [INSPIRE].

[63] D. Bardhan, P. Byakti, D. Ghosh and T. Sharma, The $750 \mathrm{GeV}$ diphoton resonance as an sgoldstino: a reappraisal, JHEP 06 (2016) 129 [arXiv:1603.05251] [INSPIRE].

[64] P. Baratella, J. Elias-Miro, J. Penedo and A. Romanino, A closer look to the sgoldstino interpretation of the diphoton excess, JHEP 06 (2016) 086 [arXiv:1603.05682] [INSPIRE].

[65] H.P. Nilles and M.W. Winkler, $750 \mathrm{GeV}$ diphotons and supersymmetric grand unification, JHEP 05 (2016) 182 [arXiv: 1604.03598] [INSPIRE]. 
[66] D. Choudhury and K. Ghosh, The LHC diphoton excess at $750 \mathrm{GeV}$ in the framework of the constrained minimal supersymmetric standard model, arXiv:1605.00013 [INSPIRE].

[67] A. Djouadi and A. Pilaftsis, The $750 \mathrm{GeV}$ diphoton resonance in the MSSM, arXiv: 1605.01040 [INSPIRE].

[68] E. Molinaro, F. Sannino and N. Vignaroli, Minimal composite dynamics versus axion origin of the diphoton excess, arXiv:1512.05334 [INSPIRE].

[69] J.M. No, V. Sanz and J. Setford, See-saw composite Higgs model at the LHC: linking naturalness to the $750 \mathrm{GeV}$ diphoton resonance, Phys. Rev. D 93 (2016) 095010 [arXiv: 1512.05700] [INSPIRE].

[70] A. Belyaev et al., Singlets in composite Higgs models in light of the LHC $750 \mathrm{GeV}$ diphoton excess, Phys. Rev. D 94 (2016) 015004 [arXiv:1512.07242] [InSPIRE].

[71] O. Antipin, M. Mojaza and F. Sannino, Minimal Coleman-Weinberg theory explains the diphoton excess, Phys. Rev. D 93 (2016) 115007 [arXiv: 1512.06708] [INSPIRE].

[72] L. Marzola, A. Racioppi, M. Raidal, F.R. Urban and H. Veermäe, Non-minimal $C W$ inflation, electroweak symmetry breaking and the $750 \mathrm{GeV}$ anomaly, JHEP 03 (2016) 190 [arXiv: 1512.09136] [INSPIRE].

[73] G.M. Pelaggi, A. Strumia and E. Vigiani, Trinification can explain the di-photon and di-boson LHC anomalies, JHEP 03 (2016) 025 [arXiv: 1512.07225] [INSPIRE].

[74] G.F. Giudice et al., Softened gravity and the extension of the standard model up to infinite energy, JHEP 02 (2015) 137 [arXiv:1412.2769] [INSPIRE].

[75] K. Kannike, Vacuum stability conditions from copositivity criteria, Eur. Phys. J. C 72 (2012) 2093 [arXiv: 1205.3781] [INSPIRE].

[76] R. Franceschini et al., Digamma, what next?, arXiv:1604.06446 [INSPIRE].

[77] ATLAS collaboration, Search for squarks and gluinos in final states with jets and missing transverse momentum at $\sqrt{s}=13 \mathrm{TeV}$ with the ATLAS detector, Eur. Phys. J. C 76 (2016) 392 [arXiv: 1605.03814] [INSPIRE].

[78] J.R. Ellis, M.K. Gaillard and D.V. Nanopoulos, A phenomenological profile of the Higgs boson, Nucl. Phys. B 106 (1976) 292 [InSPIRE].

[79] M.A. Shifman, A.I. Vainshtein, M.B. Voloshin and V.I. Zakharov, Low-energy theorems for Higgs boson couplings to photons, Sov. J. Nucl. Phys. 30 (1979) 711 [Yad. Fiz. 30 (1979) 1368] [INSPIRE].

[80] D. Buttazzo et al., Investigating the near-criticality of the Higgs boson, JHEP 12 (2013) 089 [arXiv: 1307.3536] [INSPIRE].

[81] G. Degrassi et al., Higgs mass and vacuum stability in the standard model at NNLO, JHEP 08 (2012) 098 [arXiv: 1205.6497] [INSPIRE].

[82] A. Salvio, A simple motivated completion of the standard model below the Planck scale: axions and right-handed neutrinos, Phys. Lett. B 743 (2015) 428 [arXiv:1501.03781] [INSPIRE].

[83] V. Branchina and E. Messina, Stability, Higgs boson mass and new physics, Phys. Rev. Lett. 111 (2013) 241801 [arXiv: 1307.5193] [INSPIRE].

[84] M. Dhuria and G. Goswami, Perturbativity, vacuum stability and inflation in the light of $750 \mathrm{GeV}$ diphoton excess, arXiv:1512.06782 [INSPIRE]. 
[85] J. Zhang and S. Zhou, Electroweak vacuum stability and diphoton excess at $750 \mathrm{GeV}$, Chin. Phys. C 40 (2016) 081001 [arXiv: 1512.07889] [INSPIRE].

[86] A. Salvio and A. Mazumdar, Higgs stability and the $750 \mathrm{GeV}$ diphoton excess, Phys. Lett. B 755 (2016) 469 [arXiv: 1512.08184] [INSPIRE].

[87] Y. Hamada, T. Noumi, S. Sun and G. Shiu, An O(750) GeV resonance and inflation, Phys. Rev. D 93 (2016) 123514 [arXiv:1512.08984] [inSPIRE].

[88] S.-F. Ge, H.-J. He, J. Ren and Z.-Z. Xianyu, Realizing dark matter and Higgs inflation in light of LHC diphoton excess, Phys. Lett. B 757 (2016) 480 [arXiv:1602.01801] [InSPIRE].

[89] K.J. Bae, M. Endo, K. Hamaguchi and T. Moroi, Diphoton excess and running couplings, Phys. Lett. B 757 (2016) 493 [arXiv: 1602.03653] [INSPIRE].

[90] A. Salvio and A. Mazumdar, Classical and quantum initial conditions for Higgs inflation, Phys. Lett. B 750 (2015) 194 [arXiv:1506.07520] [InSPIRE].

[91] A. Salvio, F. Staub, A. Strumia and A. Urbano, On the maximal diphoton width, JHEP 03 (2016) 214 [arXiv: 1602.01460] [InSPIRE].

[92] K. Harigaya and Y. Nomura, Composite models for the $750 \mathrm{GeV}$ diphoton excess, Phys. Lett. B 754 (2016) 151 [arXiv: 1512.04850] [INSPIRE].

[93] Y. Nakai, R. Sato and K. Tobioka, Footprints of new strong dynamics via anomaly and the $750 \mathrm{GeV}$ diphoton, Phys. Rev. Lett. 116 (2016) 151802 [arXiv: 1512. 04924] [InSPIRE].

[94] A. Pilaftsis, Diphoton Signatures from Heavy Axion Decays at the CERN Large Hadron Collider, Phys. Rev. D 93 (2016) 015017 [arXiv:1512.04931] [InSPIRE].

[95] Y. Bai, J. Berger and R. Lu, 750 GeV dark pion: cousin of a dark G-parity odd WIMP, Phys. Rev. D 93 (2016) 076009 [arXiv:1512.05779] [InSPIRE].

[96] L. Bian, N. Chen, D. Liu and J. Shu, Hidden confining world on the $750 \mathrm{GeV}$ diphoton excess, Phys. Rev. D 93 (2016) 095011 [arXiv:1512.05759] [InSPIRE].

[97] K. Harigaya and Y. Nomura, A composite model for the $750 \mathrm{GeV}$ diphoton excess, JHEP 03 (2016) 091 [arXiv: 1602.01092] [INSPIRE].

[98] M. Redi, A. Strumia, A. Tesi and E. Vigiani, Di-photon resonance and Dark Matter as heavy pions, JHEP 05 (2016) 078 [arXiv: 1602.07297] [INSPIRE].

[99] K. Harigaya and Y. Nomura, Hidden pion varieties in composite models for diphoton resonances, arXiv: 1603.05774 [INSPIRE].

[100] Y. Bai, V. Barger and J. Berger, Constraints on color-octet companions of a 750 GeV heavy pion from dijet and photon plus jet resonance searches, Phys. Rev. D 94 (2016) 011701 [arXiv: 1604. 07835] [INSPIRE].

[101] O. Antipin, M. Redi, A. Strumia and E. Vigiani, Accidental composite dark matter, JHEP 07 (2015) 039 [arXiv: 1503.08749] [INSPIRE].

[102] F. Feruglio, B. Gavela, K. Kanshin, P.A.N. Machado, S. Rigolin and S. Saa, The minimal linear $\sigma$-model for the Goldstone Higgs, JHEP 06 (2016) 038 [arXiv:1603.05668] [INSPIRE].

[103] E. Megias, O. Pujolàs and M. Quirós, On dilatons and the LHC diphoton excess, JHEP 05 (2016) 137 [arXiv: 1512.06106] [InSPIRE]. 
[104] J. Cao, L. Shang, W. Su, Y. Zhang and J. Zhu, Interpreting the $750 \mathrm{GeV}$ diphoton excess in the minimal dilaton model, Eur. Phys. J. C 76 (2016) 239 [arXiv:1601.02570] [InSPIRE].

[105] B. Agarwal, J. Isaacson and K.A. Mohan, Minimal dilaton model and the diphoton excess, arXiv: 1604.05328 [INSPIRE].

[106] R. Jinno and M. Takimoto, Probing classically conformal B-L model with gravitational waves, arXiv: 1604.05035 [INSPIRE].

[107] G. Servant, Baryogenesis from Strong CP Violation and the QCD Axion, Phys. Rev. Lett. 113 (2014) 171803 [arXiv: 1407.0030] [INSPIRE]. 